\title{
Antihypertensive Effect of Sesamin. I. Protection against Deoxycorticosterone Acetate-Salt-Induced Hypertension and Cardiovascular Hypertrophy
}

\author{
Yasuo Matsumura, ${ }^{a}$ Satomi Kita, ${ }^{a}$ Shiro Morimoto, ${ }^{*, a}$ Kengo Akimoto, ${ }^{b}$ \\ Mayumi FURUYA, ${ }^{b}$ Naomi OKA, ${ }^{b}$ and Takaharu TANAKA ${ }^{b}$ \\ Department of Pharmacology, Osaka University of Pharmaceutical Sciences, ${ }^{a}$ Kawai, Matsubara, Osaka 580, Japan \\ and Suntory Institute for Biomedical Research, ${ }^{b}$ Shimamoto, Mishima, Osaka 618, Japan.
}

Received February 20, 1995; accepted March 22, 1995

\begin{abstract}
We investigated the antihypertensive effect of sesamin, a lignan from sesame oil, using deoxycorticosterone acetate (DOCA)-salt hypertensive rats. The animals were unilaterally nephrectomized, and then separated into a sham-operated group (sham group) and a DOCA-salt-treated group. The latter was further separated into a normal diet group (control group) and a sesamin-containing diet group (sesamin group). The systolic blood pressure of control group progressively increased in comparison with that of sham group. This DOCA-salt-induced hypertension was markedly suppressed by feeding a sesamin-containing diet. Systolic blood pressure after 5 weeks was $130.6 \pm 1.9 \mathrm{mmHg}$ in the sham group, $198.1 \pm 7.3 \mathrm{mmHg}$ in the control group and $152.5 \pm 8.4 \mathrm{mmHg}$ in the sesamin group, respectively. The treatment with DOCA and salt for 5 weeks significantly increased the weight of the left ventricle plus the septum. However, this increase was significantly suppressed in the sesamin group. When the degree of vascular hypertrophy of the aorta and superior mesenteric artery was histochemically evaluated, there were significant increases in wall thickness, wall area and the wall-to-lumen ratio in the control group, compared with the sham. Sesamin feeding ameliorated the development of DOCA-salt-induced vascular hypertrophy in both the aorta and mesenteric artery. These findings strongly suggest that sesamin is useful as a prophylactic treatment in the development of hypertension and cardiovascular hypertrophy.
\end{abstract}

Key words sesamin; deoxycorticosterone-salt; hypertension; cardiac hypertrophy; vascular hypertrophy

In Asian regions, sesame seeds and sesame oil have been used widely as traditional health foods in daily life. However, little is known about the biologically active components of sesame or its biological actions. Sesamin is one of the lignans contained abundantly in sesame, but this lignan has not attracted nutritional or biological interest, unlike other lignans such as sesaminol, which is known to have antioxidative activity. ${ }^{1)}$ Recent studies have focused on the biological activities of sesamin and found that sesamin affects lipid metabolism such as desaturation in polyunsaturated fatty acid biosynthesis ${ }^{2)}$ and cholesterol absorption. ${ }^{3)}$ Most recently, it has been reported that sesamin exhibits protective effects against liver damage caused by alcohol or carbon tetrachloride, ${ }^{4)}$ and against 7,12-dimethylbenz $[a]$ anthracene-induced rat mammary carcinogenesis. ${ }^{5)}$ Thus, the biological actions of sesamin and related lignans may be multifunctional.

During our exploration of the biological activities of sesamin, we found that this compound exhibited vasorelaxing activity in the rat aortic ring (unpublished observation). This preliminary evidence led us to examine whether sesamin possesses antihypertensive activity. To attain the objective, we decided to investigate the preventive effect of sesamin in the development of experimental hypertension induced by deoxycorticosterone acetate (DOCA) and salt, which has been used extensively as a useful model of human hypertension. We report here that dietary sesamin can markedly suppress the elevation of blood pressure, cardiac hypertrophy and vascular hypertrophy in DOCA-salt hypertensive rats.

\section{MATERIALS AND METHODS}

Materials Sesamin was prepared from refined sesame oil and purified as described previously. ${ }^{6}$ Sesamincontaining diet ( $1 \mathrm{w} / \mathrm{w} \%$ in commercial normal diet) was obtained from Oriental Yeast Co., Ltd. The concentration of sesamin was determined based on the previous study. ${ }^{4)}$ All other reagents used were of analytical grade.

Animal Experiments Male Sprague-Dawley rats (6 weeks old) were anesthetized with sodium pentobarbital $(40 \mathrm{mg} / \mathrm{kg}$, i.p.) and the right kidney was removed via a right flank incision. After a 1-week postsurgical recovery period, rats were separated into a sham-operated group (sham group) and a DOCA-salt group. The latter was further separated into a normal diet group (control group) and a sesamin-containing diet group (sesamin group). The sham group fed on normal diet and tap water ad libitum. Rats of the DOCA-salt group were treated twice weekly with DOCA suspended in corn oil, which was administered subcutaneously $(15 \mathrm{mg} / \mathrm{kg})$, and $1 \% \mathrm{NaCl}$ was added to their tap water for drinking. The control group fed on a normal diet, whereas the sesamin group fed on the same diet containing $1 \%$ sesamin. Systolic blood pressure was monitored weekly with a tail cuff and a pneumatic pulse transducer (BP-98A, Softron). After 5 weeks, the animals were killed by exsanguination under anesthesia and the heart was removed. Weights of the whole heart and the left ventricle plus the septum were determined. In some rats from the three groups, the thoracic aorta and superior mesenteric artery were also removed, from which fat and adherent connective tissue were cleaned off, and they were used for morphometric analysis. 
Morphometric Analysis The thoracic aorta and superior mesenteric artery of each rat were placed in a vial of $10 \%$-formaldehyde neutral buffer solution for later analysis. Cross sections from the thoracic aorta and mesenteric arteries were cut $5 \mu \mathrm{m}$ thick and stained with Elastica-van Gieson. The vessel wall area and thickness were determined in three to four different cross sections of each vessel using a computerized digitizing system (IBAS II, Carl Zeiss, Germany).

Statistical Analysis All values were expressed as the mean \pm S.E.M. For statistical analysis, we used an analysis of variance and Duncan's multiple range test. In all comparisons, differences were considered to have statistical significance at $p<0.05$.

\section{RESULTS}

Effects of Dietary Sesamin on the Cardiac Hypertrophy of DOCA-Salt Hypertensive Rats The comparative data of cardiac hypertrophy on three groups of animals after the experimental periods of 5 weeks are summarized in Table 1. A gain in body weight of the DOCA-salt rats (control and sesamin) was smaller than that in the sham

Table 1. Comparative Data of Body Weights (BW) and Heart Weights (HW) in Sham-Operated, Control and Sesamin-Fed Rats after 5 Weeks of Treatment

\begin{tabular}{lrcc}
\hline & & & \multicolumn{2}{c}{ DOCA-salt } \\
& Sham $(n=6)$ & Control $(n=7)$ & Sesamin $(n=8)$ \\
& & & \\
BW (g) & $421.83 \pm 8.42$ & $331.43 \pm 12.23^{* *}$ & $328.75 \pm 13.85$ \\
HW (mg) & $1101.67 \pm 31.14$ & $1217.29 \pm 29.15$ & $1056.62 \pm 60.49^{\dagger}$ \\
L.V. + S. weight (mg) & $739.67 \pm 20.90$ & $932.00 \pm 22.07^{* *}$ & $779.50 \pm 43.23^{\dagger+}$ \\
HW/BW (mg/g) & $2.61 \pm 0.05$ & $3.71 \pm 0.18^{* *}$ & $3.21 \pm 0.10^{\dagger}$ \\
L.V. + S. weight/BW & $1.75 \pm 0.03$ & $2.84 \pm 0.14^{* *}$ & $2.37 \pm 0.07^{\dagger \dagger}$ \\
$(\mathrm{mg} / \mathrm{g})$ & & & \\
\hline
\end{tabular}

Values are the mean \pm S.E.M. $* *, p<0.01$, compared with sham group. $\uparrow, p<0.05 ; \uparrow+, p<0.01$, compared with control group. L.V., left ventricle; S., septum. group. There were slight and nonsignificant increases in the heart weight of the control group, compared with those of the sham group. On the other hand, the heart weight in the sesamin-fed group was significantly smaller than that in the control group. The left ventricle (L.V.) + septum weight, the heart weight-to-body weight ratio and the L.V. + septum weight-to-body weight ratio were markedly increased by the treatment with DOCA and salt for 5 weeks, but sesamin feeding produced significant lowering effects on these alterations, although the effects were incomplete.

Effects of Dietary Sesamin on the Blood Pressure of DOCA-Salt Hypertensive Rats At the beginning of the experiment, the systolic blood pressure of the sham, control and sesamin groups were $113.8 \pm 1.3,108.1 \pm 2.2$ and $107.5 \pm 2.3 \mathrm{mmHg}$, respectively. As shown in Fig. 1, blood pressure was progressively elevated by the treatment of DOCA and salt. After 2 weeks of the treatment, there were significant increases in systolic blood pressure in the control group, compared with that in the sham group, and thereafter this hypertensive effect was gradually accelerated. The elevation of blood pressure in rats fed a sesamin-containing diet was much smaller than that in the control rats, and significant lowering effects on DOCAsalt-induced hypertension were observed throughout 2 to 5 weeks. Systolic blood pressure after 5 weeks was $130.6 \pm 1.9 \mathrm{mmHg}$ in the sham group, $198.1 \pm 7.3 \mathrm{mmHg}$ in the control group and $152.5 \pm 8.4 \mathrm{mmHg}$ in the sesamin group, respectively.

Effects of Dietary Sesamin on the Vascular Hypertrophy of DOCA-Salt Hypertensive Rats Figure 2 shows typical examples of light micrographs of representative cross sections of the aorta of 3 groups of animals. The wall thickness of the aorta from control rats was greater than that from sham-operated rats. Sesamin feeding clearly ameliorated the above structural change induced by DOCA-salt. Similar results were observed with superior mesenteric arteries obtained from 3 groups (Fig. 3). The data of morphometric analysis are summarized in Table

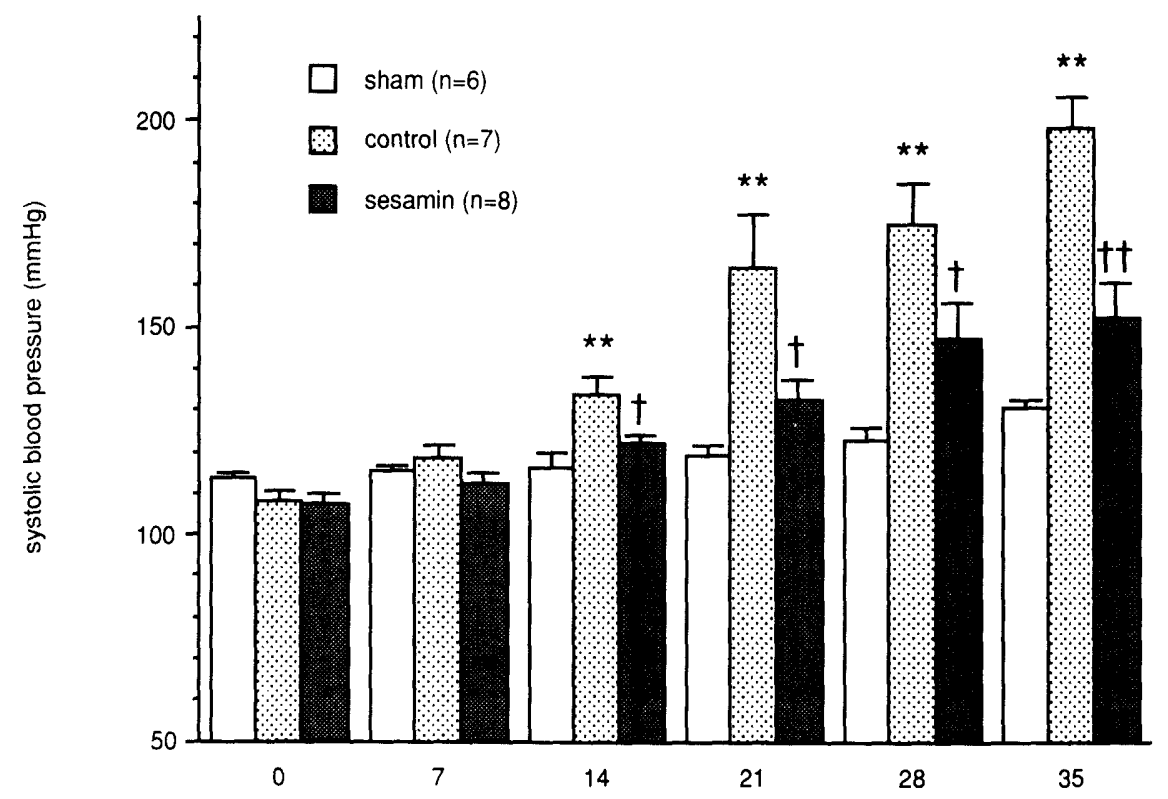

Fig. 1. Changes in Systolic Blood Pressure of Sham-Operated, Control and Sesamin-Fed Rats

Columns and bars are the mean \pm S.E.M. **, $p<0.01$, compared with sham group. $\dagger, p<0.05 ;++, p<0.01$, compared with control group. 
2. There were great increases in the wall thickness, wall area and wall-to-lumen ratio, all of which represent a degree of vascular hypertrophy, in the control group compared with the sham-operated group. Sesamin feeding significantly suppressed the above increases in all parameters of both the aorta and the mesenteric artery.

\section{DISCUSSION}

In the present study, we demonstrated that dietary sesamin efficiently suppressed the development of hypertension induced by DOCA and salt. Since sesamin feeding was started at the prehypertensive stage, our results suggest that treatment with sesamin may be a prophylactic regimen against the development of hypertension.

DOCA-salt hypertensive rats have been used extensively as a useful model of human hypertension. It has been understood that increased sympathetic nerve activity and various humoral factors, including vasopressin, play an important role in the pathogenesis of DOCA-salt hypertension. ${ }^{7)}$ Recent studies reported that the synthesis or release of endothelium-derived relaxing factor (EDRF) and responses to EDRF are impaired in this model of

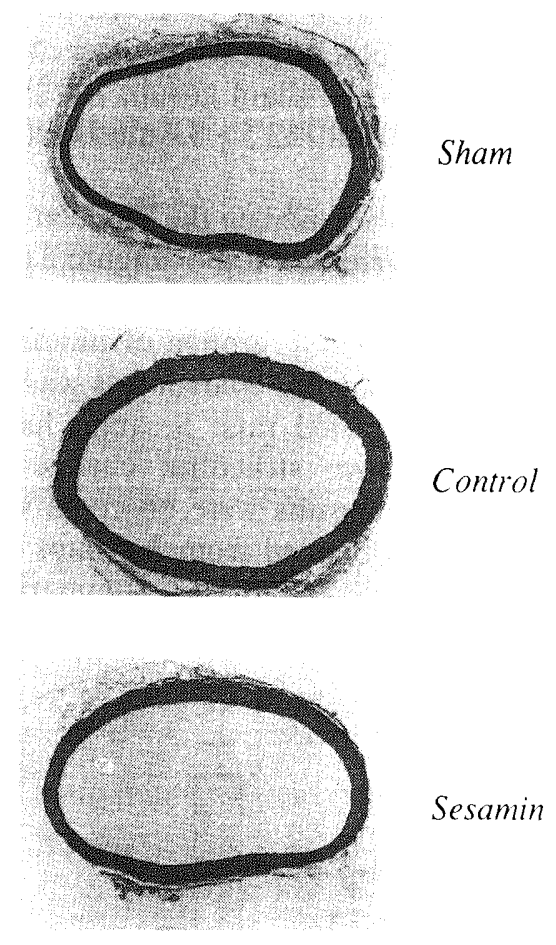

Fig. 2. Representative Light Micrographs Showing Cross Sections of Thoracic Aortas Obtained from Sham-Operated, Control and SesaminFed Rats hypertension. ${ }^{8,9)}$ In addition, we found the hypotensive effect of FR 139317, an antagonist of endothelin-1 (ET-1) receptor $\left(\mathrm{ET}_{\mathrm{A}}\right)$, in DOCA-salt hypertensive rats, thereby suggesting that ET-1 and $\mathrm{ET}_{\mathrm{A}}$ receptors are closely involved in the maintenance of DOCA-salt hypertension. ${ }^{10)}$ The mechanisms by which sesamin prevent the development of DOCA-salt hypertension are unclear, but feeding with this lignan for 5 weeks may affect the activities of the above neural and/or humoral factors. Furthermore, our preliminary experiment suggests that the vasorelaxing activity of sesamin may contribute to its antihypertensive effects. Further studies to clarify the mechanism of the antihypertensive effect of sesamin are in progress in our group.

In this study, sesamin feeding markedly suppressed cardiovascular hypertrophy in DOCA-salt hypertensive rats. It has been well acknowledged that DOCA-salt hypertension is accompanied by cardiovascular hypertrophy. ${ }^{11)}$ Since hypertension itself is a main causal factor of hypertrophy, it is possible that the blunting of the rise in blood pressure of sesamin-fed rats is associated with

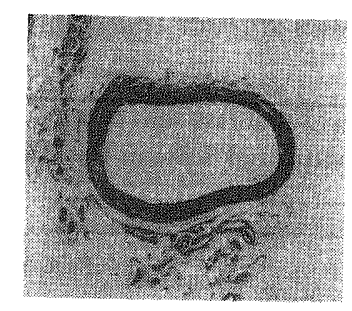

Sham

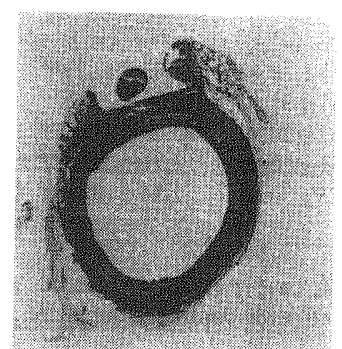

Control

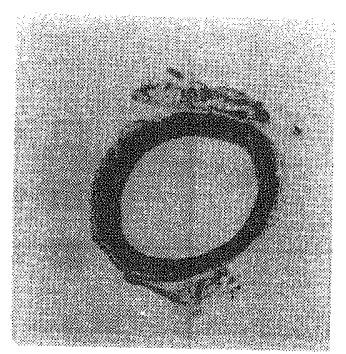

Sesamin

Fig. 3. Representative Light Micrographs Showing Cross Sections of Superior Mesenteric Arteries Obtained from Sham-Operated, Control and Sesamin-Fed Rats

Table 2. Morphological Analysis of Aorta and Mesenteric Artery in Sham-Operated, Control and Sesamin-Fed Rats after 5 Weeks of Treatment

\begin{tabular}{|c|c|c|c|c|c|c|c|}
\hline \multirow[b]{2}{*}{ Group } & \multirow[b]{2}{*}{$n$} & \multicolumn{3}{|c|}{ Aorta } & \multicolumn{3}{|c|}{ Mesenteric artery } \\
\hline & & $\begin{array}{l}\text { Wall thickness } \\
\qquad(\mu \mathrm{m})\end{array}$ & $\begin{array}{l}\text { Wall area } \\
\left(\mathrm{mm}^{2}\right)\end{array}$ & $\begin{array}{l}\text { Wall to } \\
\text { lumen ratio }\end{array}$ & $\begin{array}{l}\text { Wall thickness } \\
\qquad(\mu \mathrm{m})\end{array}$ & $\begin{array}{l}\text { Wall area } \\
\left(\mathrm{mm}^{2}\right)\end{array}$ & $\begin{array}{l}\text { Wall to } \\
\text { lumen ratio }\end{array}$ \\
\hline Sham & 4 & $95 \pm 1$ & $0.41 \pm 0.01$ & $0.27 \pm 0.02$ & $56 \pm 1$ & $0.12 \pm 0.01$ & $0.33 \pm 0.02$ \\
\hline DOCA-salt (control) & 3 & $136 \pm 5^{* *}$ & $0.63 \pm 0.04 * *$ & $0.35 \pm 0.02 * *$ & $89 \pm 2 * *$ & $0.24 \pm 0.01^{* *}$ & $0.49 \pm 0.02 * *$ \\
\hline DOCA-salt (sesamin) & 5 & $107 \pm 4^{\dagger+}$ & $0.48 \pm 0.01^{\dagger \dagger}$ & $0.28 \pm 0.01^{\dagger \dagger}$ & $73 \pm 3^{+\dagger}$ & $0.16 \pm 0.01^{\dagger \dagger}$ & $0.42 \pm 0.02^{\dagger}$ \\
\hline
\end{tabular}

Values are the mean \pm S.E.M. $\quad *, p<0.05 ; * *, p<0.01$, compared with sham group. $\dagger, p<0.05 ; \dagger \uparrow, p<0.01$, compared with control group. 
the absence of cardiovascular hypertrophy. On the other hand, a previous study ${ }^{12}$ using one-kidney, one-clip hypertensive rats demonstrated that the attenuation of vascular hypertrophy was observed after captopril treatment at a dose which did not lower blood pressure effectively. Moreover, vasorelaxing agents such as hydralazine failed to suppress vascular hypertrophy, even at a hypotensive dose. ${ }^{13)}$ These observations suggest that factors other than blood pressure per se are involved in cardiovascular hypertrophy. In order to clarify whether sesamin selectively prevents against cardiovascular hypertrophy, the effects of a lower dose or non-hypotensive dose of sesamin should be examined.

Recently, biologically beneficial effects of sesamin have been reported. Shimizu et al. $^{2)}$ reported that sesamin interfered with the metabolism of linoleic acid at the step catalyzed by 45 -desaturase in microorganisms. If such a phenomenon occurs in mammalian cells, there is a possibility that the production of prostaglandins (PGs) such as $\mathrm{PGE}_{1}$ from dihomo- $\gamma$-linolenic acid would be stimulated. Actually, $\mathrm{PGE}_{1}$ is reported to prevent the development of medial hypertrophy occurring in chronic hypoxia. ${ }^{14)}$ Hirose et al. ${ }^{3)}$ noted that sesamin lowered both serum and liver cholesterol levels by inhibiting the absorption and synthesis of cholesterol, thereby suggesting that sesamin may be an efficient hypocholesterolemic agent. Most recently, it has been reported that sesamin exhibits protective effects against liver damage caused by alcohol or carbon tetrachloride $e^{4)}$ and against 7,12dimethylbenz $[a]$ anthracene-induced rat mammary carcinogenesis. ${ }^{\text {s) }}$
In the present study, we demonstrated for the first time that sesamin efficiently prevents the development of hypertension and concomitant cardiovascular hypertrophy. The possibility that sesamin is useful as a protective agent against hypertension at the clinical level warrants further attention.

\section{REFERENCES}

1) Osawa T., Nagata M., Namiki M., Fukuda Y., Agric. Biol. Chem., 49, 3351 (1985)

2) Shimizu S., Akimoto K., Shinmen Y., Kawashima H., Sugano M., Yamada H., Lipids, 26, 512 (1991).

3) Hirose N., Inoue T., Nishihara K., Sugano M., Akimoto K., Shimizu S., Yamada H., J. Lipid Res., 32, 629 (1991).

4) Akimoto K., Kitagawa Y., Akamatsu T., Hirose N., Sugano M., Shimizu S., Yamada H., Ann. Nutr. Metab., 37, 218 (1993).

5) Hirose N., Doi F., Ueki T., Akazawa K., Chijiiwa K., Sugano M., Akimoto K., Shimizu S., Yamada H., Anticancer Res., 12, 1259 (1992).

6) Fukuda Y., Nagata T., Osawa T., Namiki M., J. Am. Oil Chem. Soc., 63, 1027 (1986).

7) Gavras H., Gavras I., J. Hypertens., 7, 601 (1989).

8) Kirchner K. A., Scanlon Jr. P. H., Dzielak D. J., Hester R. L., Am. J. Physiol., 265, R568 (1993).

9) Hayakawa H., Hirata Y., Suzuki E., Kimura K., Kikuchi K., Nagano T., Hirobe M., Omata M., Hypertension, 23, 752 (1994).

10) Fujita K., Matsumura Y., Kita S., Miyazaki Y., Hisaki K., Takaoka M., Morimoto S., Br. J. Pharmacol., 114, 925 (1995).

11) Li J. S., Lariviere R., Schiffrin E. L., Hypertension, 24, 183 (1994).

12) Wang D-H., Prewitt R. L., Hypertension, 15, 68 (1990).

13) Owens G. K., Circ. Res., 56, 525 (1985).

14) Kashani I. A., Richardson B., Lammers R. J., Merritt T. A., Bloor. C. M., Angiology, 37, 21 (1986). 